\title{
Removing Inefficiencies from Scientific Code: the Study of the Higgs Boson Couplings to Top Quarks
}

\author{
André Pereira ${ }^{1,2}$, António Onofre ${ }^{1,2}$, and \\ Alberto Proença ${ }^{1}$ \\ 1 Universidade do Minho, Portugal \\ ${ }^{2}$ LIP-Minho, Portugal \\ \{ampereira, aproenca\}@di.uminho.pt,onofre@fisica.uminho.pt
}

\begin{abstract}
This paper presents a set of methods and techniques to remove inefficiencies in a data analysis application used in searches by the ATLAS Experiment at the Large Hadron Collider. Profiling scientific code helped to pinpoint design and runtime inefficiencies, the former due to coding and data structure design. The data analysis code used by groups doing searches in the ATLAS Experiment contributed to clearly identify some of these inefficiencies and to give suggestions on how to prevent and overcome those common situations in scientific code to improve the efficient use of available computational resources in a parallel homogeneous platform.
\end{abstract}

Keywords: Scientific Computing, High Performance Computing, Code Efficiency, ATLAS Experiment

\section{Introduction}

At the European Organization for Nuclear Research (CERN), the fundamental structure of the universe is studied using the most complex scientific instruments built by physicists and engineers up to now. CERN was founded in 1954 by 12 members states but has grown to the size of a world lab with 21 member states and more then 30 states to which were given the status of "observer" states. The instrumentation used in nuclear and particle physics research is essentially formed by particle accelerators and detectors. The Large Hadron Collider (LHC) speeds up groups of particles close to the speed of light, in opposite directions, inducing a controlled collision of protons at the detectors core. The detectors record various characteristics of the resultant particles of each collision (an event), such as energy and momentum, which originate from complex decay chains of particles produced in the interaction of the partons inside the colliding protons. The purpose of these experiments is to test models and predictions in High Energy Physics (HEP), such as the Standard Model, by confirming or discovering new particles and interactions.

The ATLAS Experiment [1] is one of the seven particle detectors at the LHC. ATLAS goals are to study the properties of the recently discovered Higgs 
Pereira, Onofre, and Proença

boson [2], the search for new particles predicted by models of physics beyond the Standard Model like Susy, searches for new heavy gauge bosons and precision measurements where the top quark is of utmost importance. Approximately 600 million collisions occur every second at the LHC. Particles produced in head-on proton collisions interact with the detectors, generating massive amounts of raw data. It is estimated that all the combined detectors produce 25 petabytes of data per year, and it is expected to grow after the ongoing LHC upgrade [3]. This data then passes a set of processing and reconstruction stages until it is ready to be used by specific analysis codes developed to search for interesting events predicted by several HEP models that may be present in data. Several research groups work in event reconstruction in the same experiment, enforcing positive competition to produce quality results in a fast and consistent way.

These factors enforce the need to process more data, more accurately, in less time, which often leads to investments on larger computing clusters to improve the quality of the research results. However, most scientific code was not designed and/or developed for an efficient use of the available computational resources. If these applications were adequately designed (or tuned), the event analysis throughput could be massively increased. An efficient parallel application can significantly improve its performance at a much lower cost [4].

This paper addresses inefficiencies in two stages of the data analysis application: the code development and application runtime. In the former, inefficiencies in the algorithm coding and data structuring are pinpointed and several solutions are suggested, based on a quantitative analysis of the bottlenecks. The latter identifies inefficiencies in threads accessing remote shared memory, and gives hints to overcome these limitations.

This paper is organized as follows: section 2 briefly presents the top quark and Higgs boson decay process and introduces a short characterization of the data analysis application used as case study; in section 3 the code inefficiencies are identified, analysed, and removed, with a final shared memory parallelization proposal; in section 4, runtime inefficiencies of the parallelization are identified and possible alternatives suggested, concluding with an assessment of the core affinity impact; finally, section 5 concludes the paper with suggestions for future work.

\section{Top Quark and Higgs Boson Decay}

At the LHC, two proton beams are accelerated close to the speed of light in opposite directions, set to collide inside a specific particle detector. This headon collision triggers a chain reaction of decaying particles, and most of the final particles interact with the detector, allowing to record relevant data. One of the searches being conducted at the ATLAS Experiment relates to the study of the top quark and Higgs boson couplings. Figure 1 represents the final state topology of the associated production of two top quarks and one Higgs boson (that decays to two b-quarks), labelled from now on as $t \bar{t} H$ production. 


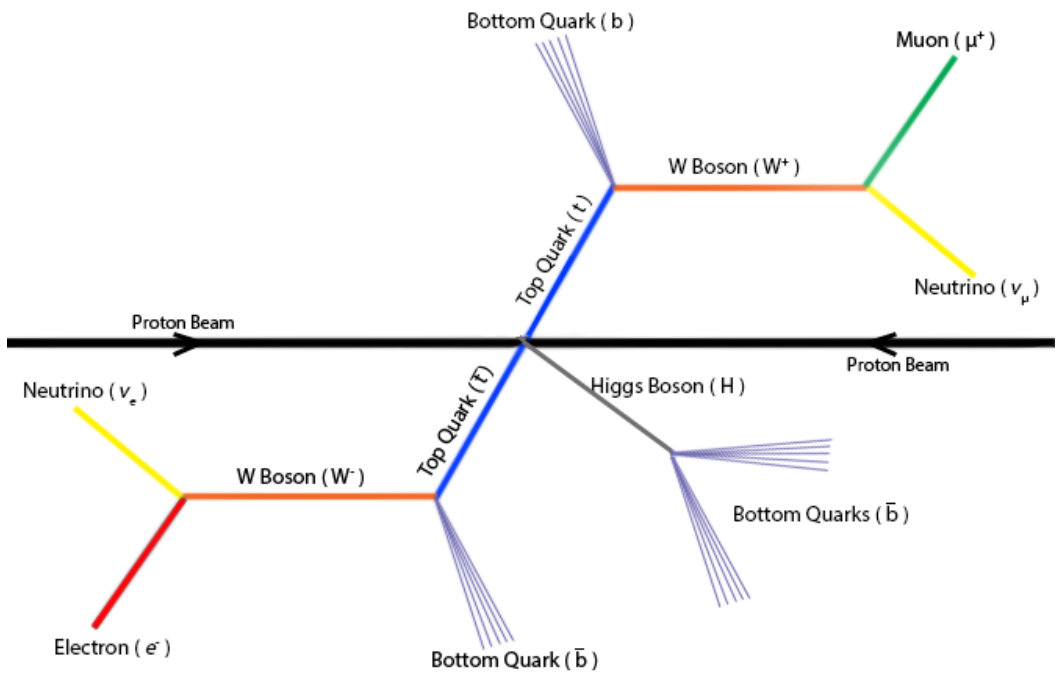

Fig. 1: Schematic representation of the $t \bar{t}$ system and Higgs boson decay.

The ATLAS detector can record the characteristics of the bottom quarks, detected as a jet of particles, and leptons (muon and electron). Neutrinos do not interact with the detector, so, their characteristics are not recorded. Since the top quark reconstruction requires the neutrinos, their characteristics are analytically determined with the known information of the system, through a kinematical reconstruction. However, the $t \bar{t}$ system may not have a possible reconstruction: the reconstruction has an intrinsic uncertainty associated which determines its accuracy.

The amount of jets from bottom quarks and leptons present in the events may vary according to the decay channel of the $\mathrm{W}$ bosons produced in the top quark decays. As shown in figure 1, four jets and two leptons are required to be present in the events. Two of the jets, together with two leptons are required to reconstruct the $t \bar{t}$ system, and the remaining two jets are used for the Higgs boson reconstruction. For the kinematical reconstruction, every possible combination of jets and leptons must be evaluated and only the most accurate reconstruction of each event is considered. In a first step, the $t \bar{t}$ system reconstruction is tried. If it has a possible solution, the Higgs boson is reconstructed from the jets of the two remaining bottom quarks. The Higgs reconstruction does not use the jets which were associated to the best $t \bar{t}$ system reconstruction. The overall quality of the event processing depends on the quality of both reconstructions.

For the global event reconstruction, several solutions can be tested if we assume that the ATLAS detector has an experimental energy-momentum resolution of $\pm 1 \%$, by varying these quantities within their uncertainty. This uncertainty is propagated into the $t \bar{t}$ system and Higgs reconstructions, affecting their 
accuracy. To improve the quality of the reconstructions several random variations are applied to the measured values, within a maximum range of $|1 \%|$ next to the measured values. The quality of the reconstructions and the application execution time is directly proportional to the amount of variations performed per combination. The goal is to do as many variations as possible within a reasonable time frame.

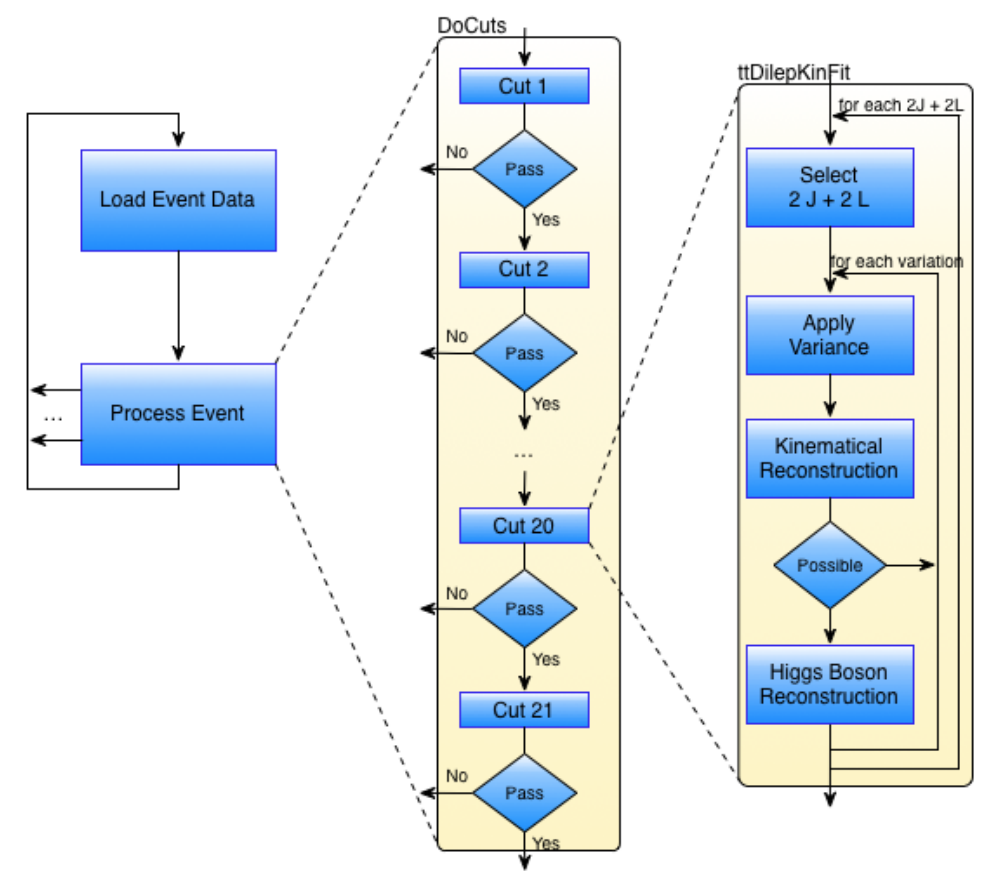

Fig. 2: Schematic representation for the ttH_dilep application flow.

To reconstruct the $t \bar{t} H$ system a data analysis application was developed, the $t$ tH_dilep. The application flow is presented in figure 2. Each event data on an input file is individually loaded into a single global state, shared between the data analysis code and the LipMiniAnalysis toolbox ${ }^{3}$, and it is overwritten every time a new event is loaded. The event is then submitted to a series of cuts, which filters events that are not suited for reconstruction. When an event reaches the cut 20, the $t \bar{t}$ system and Higgs boson are reconstructed in the function ttDilepKinFit, which is expected to be the most computing demanding. If the $t \bar{t}$ system reconstruction fails, the current combination is discarded and the next

\footnotetext{
${ }^{3}$ The LipMiniAnalysis toolbox provides a skeleton to several data analysis applications under study in the Portuguese LIP institution, a CERN partner in the ATLAS Experiment.
} 
is processed. If an event has a possible reconstruction it passes the final cut and its final information is stored.

The application also depends on the ROOT framework ${ }^{4}$ for part of the functionalities used in the reconstructions and for result output and visualisation. The code from both ROOT and the LipMiniAnalysis toolbox cannot be modified as many data analysis applications depend on them.

\section{$3 \quad$ Coding Inefficiencies}

Inefficiency removal is a two stage iterative process, where bottlenecks are identified and later removed. First, the application is profiled and analysed to identify the critical sections of the code that take longer to compute. Then, the critical section is optimized by modifying the code, algorithm, or parallelization. The identification of critical sections can be automated by using third party tools, such as gprof [6], Callgrind [7], or VTune [8], which produce reports listing the percentage of time spent in each of the application functions. A more detailed analysis can be obtained using tools similar to PAPI [9], where hardware counters are used to quantify cache miss rates, executed floating point instructions, and other low level information.

The test environment used in both this section and section 4 is a dual-socket system with two Intel Xeon E5-2670v2 [10] with 10 cores, with hardware support for 20 simultaneous threads, at $2.5 \mathrm{GHz}$ each, $256 \mathrm{~KB}$ L2 cache per core and 25 MB shared L3 cache, with 64 GB DDR3 RAM. The K-Best measurement heuristic ${ }^{5}$ was adopted to ensure that the only the best, but consistent, time measurements are considered. Software wise, the GNU Compiler version 4.8.2 with $O 3$ optimizations enabled and ROOT 5.34/17 were used. A 5\% interval was used for a $k$ of 4 , with a minimum of 12 and maximum of 24 time measurements.

Profiling the data analysis code using Callgrind, the ttDilepKinFit was identified as the most time consuming function, taking $99 \%$ of the execution time for 1024 variations. ttH_dilep execution with this amount of variations was considered reasonable for all efficiency measurements unless stated otherwise, without compromising the application execution time.

A preliminary computational analysis concluded that the application is compute bound on the testbed system, where accesses to the system RAM memory are not a limiting factor with a ratio of 7 instructions per fetched byte for 1024 variations.

An analysis of the code showed two major inefficiencies restricting the performance: (i) the pseudo-random number generation is consuming a large part of the ttDilepKinFit execution time, (ii) the way data is structured in the

\footnotetext{
${ }^{4} \mathrm{ROOT}[5]$ is a $\mathrm{C}++$ framework produced by CERN to help the development of particle data analysis code, by implementing specific features.

${ }^{5}$ For a detailed explanation of the K-Best Measurement Scheme, see Chapter 9.4.3 of Computer Systems: A Programmer's Perspective (CS:APP), Randal E. Bryant and David R. O'Hallaron, Prentice Hall, 2003.
} 
LipMiniAnalysis prevents processing in parallel events from the same input file. These two issues are further detailed in the next subsections.

\subsection{Pseudo-Random Number Generation Inefficiencies}

Pseudo-random number generators (PRNGs) are common in many Monte Carlo simulation and reconstruction applications. A good PRNG deterministically generates uniform numbers with a long period, its produced values pass a set of randomness tests and, in HPC, it must be efficient and scalable. Repeatability is ensured by providing a seed to the PRNG prior to number generation, due to their deterministic execution.

The reconstruction of $t \bar{t} H$ events depends crucially in the intrinsic energymomentum resolution of the ATLAS Experiment. In order to increase the probability of finding the correct solution for the particular configuration of the $t \bar{t} H$ event under study, pseudo experiments are performed. For each pseudo experiment, the event kinematics is varied by applying an offset which changes the energy-momentum four vectors of final state particles, within detector resolutions. As a case study, the maximum offset has been set to $\pm 1 \%$ of the original value and is computed with the help of PRNG. An analysis of the callgraph produced for 256 variations (higher variations made the Callgrind execution time infeasible) showed that $63 \%$ of $\mathrm{ttH}$ _dilep execution time was spent on the PRNG. However, $23 \%$ of the time was spent defining a new seed for the PRNG. Figure 3 presents the callgraph for the ttDilepKinFit function of $t$ tH_dilep.

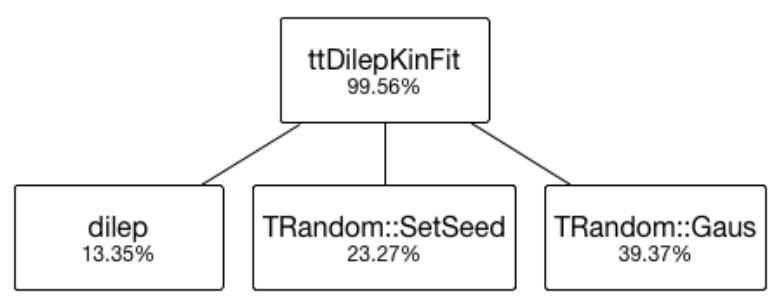

Fig. 3: Callgraph subset of the ttDilepKinFit most time consuming functions for 256 variations per combination.

An analysis of the code showed that the application uses a PRNG available in ROOT, which uses the Mersenne Twister algorithm [11], resetting the seed for every parameter variation. The Mersenne Twister period is approximately $4.3 *$ $10^{6001}$, while the maximum amount of pseudo random numbers generated by the application, for the input file used and 1024 variations, is $3 * 10^{9}$, making the seed reset unnecessary. The removal of this inefficiency granted a $71 \%$ performance improvement. 


\subsection{Data Structure Inefficiencies}

Once removed the PRNG seed reset inefficiency, the ttDilepKinFit still remained the critical region in the application, with no apparent code inefficiency. The most obvious solution is to process in parallel several events from the same input file. However, the function in LipMiniAnalysis that loads events from a file into memory assigns a single global space. This data structure contains information that is modified during the event reconstruction process, and it is overwritten for every event loaded. Changing the data structure to support multiple events in memory simultaneously, and loading all events in the input file at the beginning of the data analysis, would allow the parallel processing of events with low overhead. However, as mentioned in section 2 many data analysis applications depend on LipMiniAnalysis preventing any modifications to its structure, so alternative solutions were explored.

\subsection{Alternative Parallel Approaches}

Next step to improve the code execution time is to parallelize ttDilepKinFit. Note that it is not possible to parallelize the whole event processing since only one is loaded at a time and part of its information is stored in LipMiniAnalysis toolbox. Besides not allowing this parallelization, reading events individually is more inefficient than reading all events at once, where in the former slower random reads are made on the hard drive and in the latter the fast sequential reads are used.

Parallelizing ttDilepKinFit implies modifying its flow. Currently, for each different combination of jets and leptons from an event, the processed data of each variation of the detector measurements is overwritten. A new data structure is required to hold all combinations of each event. Picking a lepton/jet combination depends on all previous chosen combinations, which serializes the construction of the data structure. Each parallel task (indivisible work segment) selects a combination with variations still to compute, then varies the particles parameters, performs the kinematical reconstruction, and attempts to reconstruct the Higgs boson. A parallel merge is performed after all combinations are computed to get the most accurate reconstruction for the event. Figure 4 presents the sequential and parallel workflow for ttDilepKinFit.

A shared memory parallelization using OpenMP [12] was devised, as it is the best approach for single shared memory systems. The parallel tasks are grouped into threads, which holds the best reconstruction to minimize the complexity of the merge by reducing through all the threads instead of tasks. The amount of tasks for each thread is balanced dynamically by the OpenMP scheduler, as the workload is irregular since the Higgs boson reconstruction execution is not always computed. Each thread has a private PRNG initialized with different seeds to avoid correlation between the numbers generated.

Figure 5 presents the speedups for different number of parallel threads. The purpose of the 1 thread test is to evaluate the parallelization overhead. The best efficient implementation occurs when using 2 and 4 threads, where the 
Pereira, Onofre, and Proença

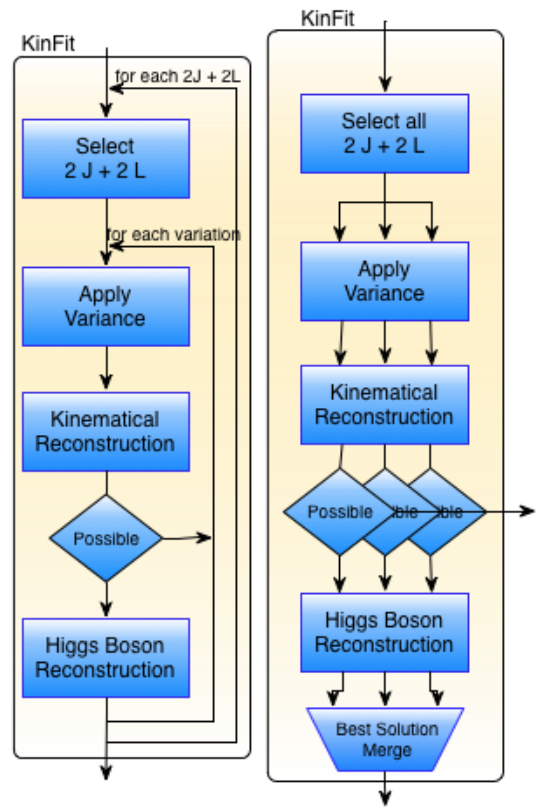

Fig. 4: Schematic representation of the ttDilepKinFit workflows: sequential (left) and parallel (right).

application is using almost all resources at each used core. The best overall performance occurs for 40 threads, but it only offers a speedup of 8.8 , underusing the available 20 physical cores. Note that there is no significant overhead due to NUMA $^{6}$ accesses, as seen by the constant increase in performance from 10 to 16 threads. For more than 20 threads all available resources on both CPU devices.

The lack of scalability beyond a low number of parallel threads suggests that inefficiencies may still affect the application, probably caused by the parallelization overhead. Intel's VTune was used to search for hotspots (bottlenecks) on the parallel ttH_dilep, since this tool is best suited for profiling parallel applications while providing a user friendly graphic interface. A preliminary analysis showed that the application was spending $20 \%$ of the execution time building the combination data structure for 256 variations.

An analysis of the coded data structure showed that inefficiencies were affecting the performance in specific situations. Data that is read-only on the parallel section is being replicated in each element of the data structure. If the elements were to share a pointer to such data, the overhead of constructing the data structure would be reduced. However, this could lead to worse cache management,

\footnotetext{
${ }^{6}$ NUMA, Non-Unified Memory Access, since each Xeon device has its own memory controller with attached RAM: RAM access time for each core differs as the RAM is connected to the same device or the neighbour Xeon.
} 
Removing Inefficiencies From Scientific Code

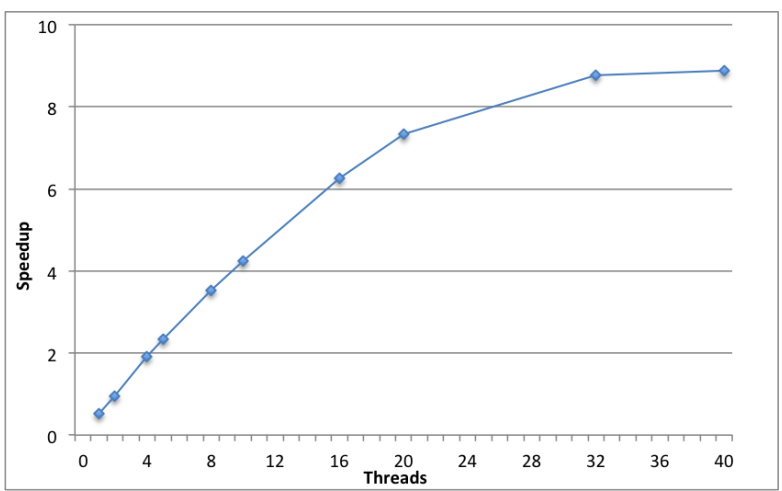

Fig. 5: Speedup for the ttH_dilep original parallel version of the application.

due to cache line invalidations, since the application is accessing data on memory more frequently, and the data structuring did not efficiently separate read-only data from read/write data. This is particularly critical in NUMA environments, where communication costs are higher. This was implemented and tested (addressed as pointer version), with its speedups plotted in figure 6 . The reference value for the speedup computation is still the same sequential version.

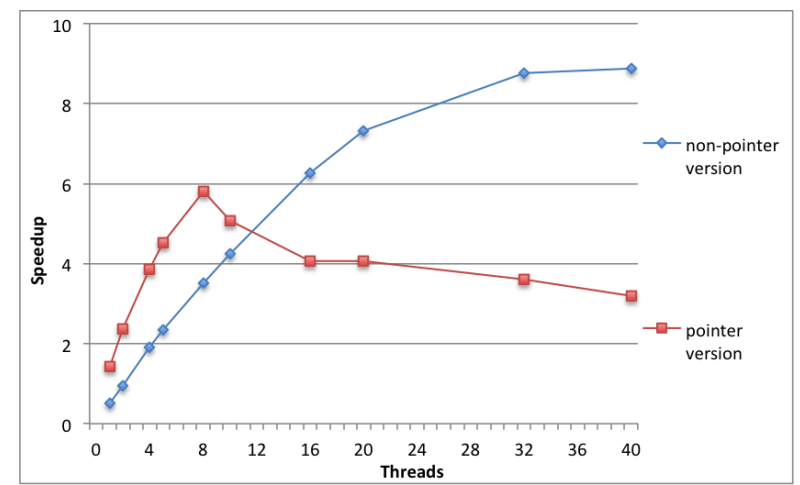

Fig. 6: Speedup for ttH_dilep parallel pointer and non-pointer implementations.

As expected, the best speedup occurs when using only one CPU device. The performance degradation from 8 to 10 threads (on the same device) may be explained by the increase of concurrent accesses to the shared L3 cache. However, this implementation is more efficient than the non-pointer implementation when using only one device. This is a classical case where partitioning the data structure allows it to fit on the L2 cache, providing superscalar speedups for certain 
Pereira, Onofre, and Proença

number of threads. The data structure has an average size of $30 \mathrm{~KB}$, but can have up to $867 \mathrm{~KB}$ with the input used. Considering the 5 thread superscalarity, partitioning the data structure among 5 cores reduces its size per core to 173 $\mathrm{KB}$, fitting their private L2 cache and avoiding the slower accesses to the L3 cache.

\section{Runtime Inefficiencies}

When submitting a job or application for execution on a given computing system, most users trust the default configurations of the submission environment. However, if the user needs to improve the efficiency of the code execution, he/she must be aware of the environment variables that can be controlled and how those can impair the performance. Two cases will be addressed here: (i) how to spread the code parallelism, between processes and threads, and (ii) how to allocate the available cores on each device to threads and processes.

\subsection{Multithreading Inefficiencies}

Without the sensibility provided by the tests in section 3 , a scientist would incur in the pitfall of using all available cores on the system (and even all hardware threads, if each core supports hardware multithreading), hoping that it would provide the best performance. While it may be true for the non-pointer implementation, the system computational resources would be inefficiently used, and using the single device highly efficient pointer implementation would induce a even greater waste.

A closer look to the pointer based implementation shows in fact that it is the most efficient one. As seen in section 3.2, the scalability of the parallelization is limited by the NUMA organization on modern multiple CPU device systems. If the threads on $c p u_{1}$ do not share information with the threads on $c p u_{2}$, the NUMA bottleneck is removed by using multithreaded processes. However, parallelization at the process level, where each process performs a data analysis on a separate event, is not possible with the current implementation of LipMiniAnalysis, where a single global state is allocated to store data from each event processing.

Data analysis applications are individually executed for each file (around 1GB in size) in a very large set of files, at a terabyte scale, received weekly from CERN. An alternative approach to the process parallelization over a single input data file is to balance the execution of different $t t H_{-} d i l e p$ processes in the system on a set of distinct input files. This reduces the complexity of the implementation, with no changes needed for ttH_dilep, and avoids communication between processes. A simple scheduler was devised, which takes a set of input files and spawns a given amount of ttH_dilep processes. The scheduler dispatches the files to the different processes in a queue-like approach, and monitor their execution as shown in figure 7 . A set of 20 input files was considered for testing and evaluation purposes, with different configurations of processes and threads per process. 


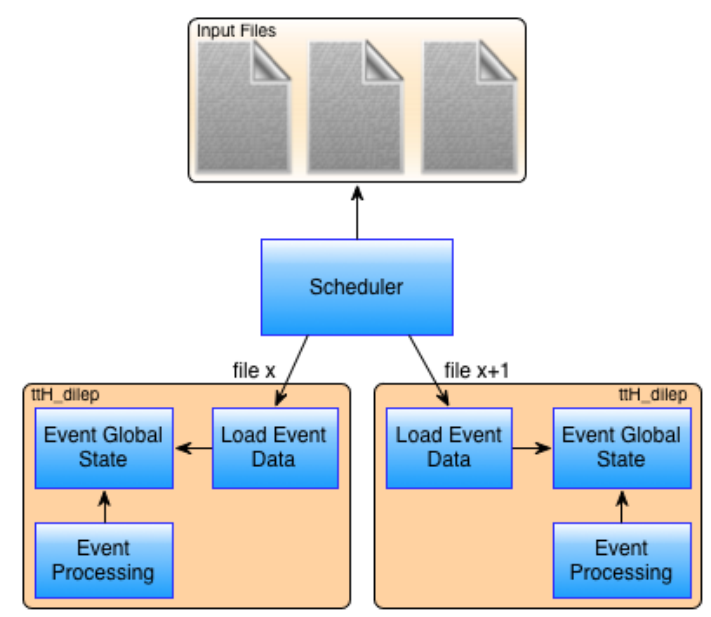

Fig. 7: Schematic representation of dispatcher workflow.

Figure 8 presents the speedups using 2, 4, 5, 8, and 10 processes for various thread configurations, with maximum number of threads limited to 40 . A higher amount of processes was not tested as the efficiency decayed from 8 to 10 processes. The best speedups occur for 8 processes with 5 threads each, with a peak of $69.3,7.8$ and 11.7 times better than the best non-pointer and pointer implementations, respectively. A small number of threads such as this allows for a small overhead in the $t t H \_d i l e p$ parallelization, namely on load balancing and final best reconstruction merge for each event. For 10 processes the load on the system due to the lack of shared memory and I/O operations affects the performance, decreasing the speedups relatively to using 8 processes. A common behaviour is that when using the CPU devices hardware multithreading the speedups tend to stabilize, or even drop for 2, 4, and 5 processes. Overall, the best speedups occur when using all available cores on the system, with multithreading.

Since this implementation uses the parallel pointer version, which has superlinear speedups as presented at the end of subsection 3.3, the performance improvements tend to be higher than the theoretical maximum. Here, the superscalarity is enhanced because the data structure is less partitioned than with the pointer version for the same number of threads, as threads from the same process are paired in each core to allow sharing common data on the L2 cache.

\subsection{Core Affinity Inefficiencies}

One of the key issues in runtime efficiency is the thread affinity [13], namely to control the allocation of each thread to which CPU core. By default, OpenMP lets the operating system to manage the thread affinity; as a consequence, threads may migrate among cores during runtime. If a thread is running on core $c_{1}$ and 
Pereira, Onofre, and Proença

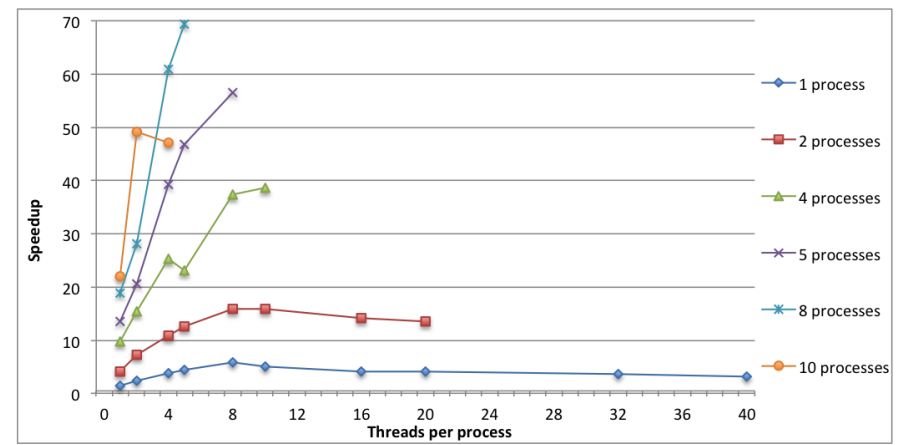

Fig. 8: Speedups for the scheduler with the pointer based implementation for several combinations of \#processes and \#threads per process.

moves to core $c_{2}$, all data on the private cache $l_{c_{1}}$ needs to be reloaded to cache $l_{c_{2}}$, causing unnecessary overhead. This effect is amplified if the threads are moved between adjacent CPU devices. When multiple different, and (possibly) parallel processes are running on the same system, which is common in production environments, such scheduling occurrences happen more frequently. This subsection presents a preliminary study on thread affinity for data analysis code.

Defining the thread affinity of an application may provide a more predictable, or in some cases better, performance. In theory, an optimum thread affinity scheme allocates the threads to contiguous physical cores of one CPU device, uses the cores of the second CPU device only after the first is filled, and finally uses the multithreading capability after filling all physical cores. Note that using multithreading before the second CPU device is fully occupied may provide better performance in memory bound applications. This type of affinity must be defined prior to the application execution and depends on the system used. In this compute bound data analysis case, the affinity was specifically tuned to the 20-core testbed system for all threads or process/threads configurations for the scheduler.

By analysing the speedups of the pointer implementation of ttH_dilep with thread affinity, in figure 9, the specification of the affinity provides speedups for the previous most efficient number of threads, i.e., up to 8 threads. For 8 threads the performance increases by $41 \%$, relative to its no affinity counterpart. With this number of threads, and the amount of shared data, moving threads between cores at runtime causes more cache warm ups to occur, significantly affecting the performance. When using more than 10 threads the application is roughly $4 \%$ slower as the operating system uses some multithreaded cores rather than using all available physical cores and it does a better job at managing the multithreading.

The same affinity study was performed on the scheduler, with the speedups presented in figure 10. The performance is increased for some specific configura- 
Removing Inefficiencies From Scientific Code

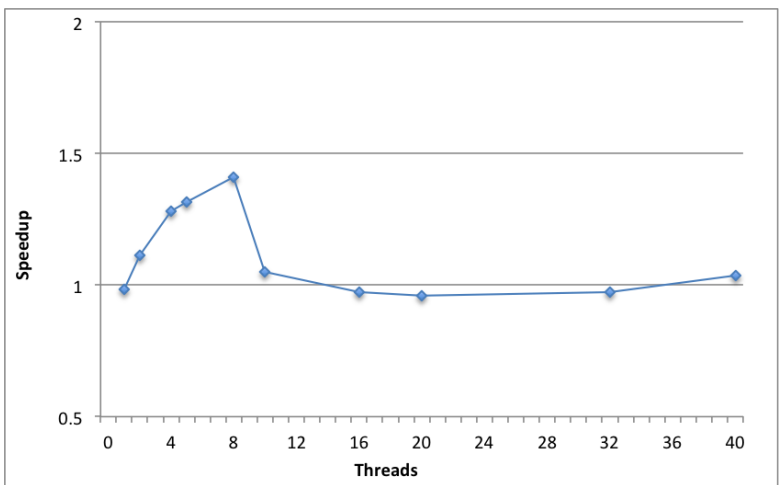

Fig. 9: Speedup of the ttH_dilep parallel pointer implementation with core affinity.

tions, with the exception of 5 processes that is always worse, providing improvements up to $52 \%, 90 \%, 8 \%$, and $25 \%$ for $2,4,8$, and 10 processes respectively.

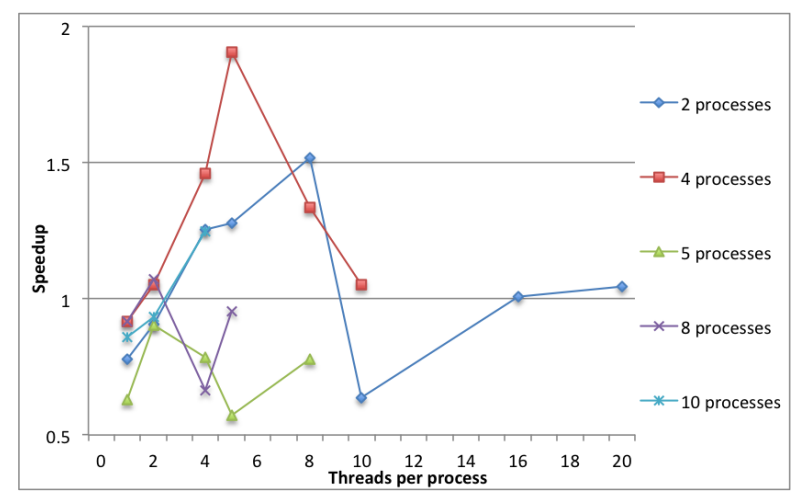

Fig. 10: Speedups of the scheduler with the pointer based implementation for various threads per process with core affinity.

The performance with core affinity is less susceptible to oscillations, as with no affinity it is sometimes affected by OS thread reallocations. It is when many reallocations may occur that setting the core affinity provides the best performance. Hard setting the affinity may not allow for proper multithreading to hide the memory accesses latency, affecting the performance. It is not possible to use a theoretical affinity scheme to always improve the performance on every system, as it is highly dependent on: 
Pereira, Onofre, and Proença

- the algorithm, memory bound, suffers more from core reallocation due to losing all data on cache, where fixing their position on a specific core avoids unnecessary accesses to the RAM;

- the application execution time, as the impact from thread reallocations is higher in applications with low execution times;

- the operating system, as OpenMP, by default, lets it manage the thread allocation and it is susceptible to the overall system load, causing fluctuations in consecutive applications execution time.

With all optimizations considered, the best overall performance in a dual 10-core Xeon system is obtained using the scheduler combined with the pointer implementation, with 8 multithreaded processes (with 5 threads each), reaching a speedup of 112 over the original sequential application.

\section{Conclusion}

This paper presents a study of the inefficiencies in scientific code, using a particle reconstruction analysis application as a case study. Top quark and Higgs boson studies require reconstructing from measurements of a very large number of particle collisions, performed weekly by the ttH_dilep application on terabytes of data. A faster and more accurate analysis of the data allows to better reconstruct $t \bar{t} H$ events and improve the quality of the research results.

Execution inefficiencies of software applications may occur due to several factors, from algorithm and data structure design to numerical approaches, choice of library functions or compile tuning for code vectorisation, among others. Our focus in this work was on the identification and removal of inefficiencies at only two stages of the application - the code design and its submission for execution - since efficient numerical libraries were already used [14], as well as the Intel guidelines to develop efficient code [15-17]. The code inefficiencies we identified and corrected had a significant negative impact on performance: the removal of the unnecessary seed generation for the pseudo random number generation led to $71 \%$ performance improvement. Two parallelisation alternatives were proposed to overcome data structure inefficiencies, one that scales with two CPU devices and other much more efficient but only scales with one CPU device.

At application runtime, a multiprocess approach using the more efficient parallel implementation tackled its inefficiencies on NUMA systems, providing a superscalar speedup of 69.3 with 40 threads. This superscalarity was achieved due to the data partition, which led to a better cache usage. An efficient control on the thread affinity of this implementation provided a performance improvement close to $2 \mathrm{x}$. However, the fluctuation in performance and the dependencies on many system characteristics prevented the definition of a generalized heuristic to aid to control the best affinity for the application, for any computing system.

Figure 11 plots the number of events processed per second in four different situations using a dual 10-core Xeon computing system: (i) assuming the particle properties as given by the ATLAS detector with $100 \%$ confidence, (ii) the same 


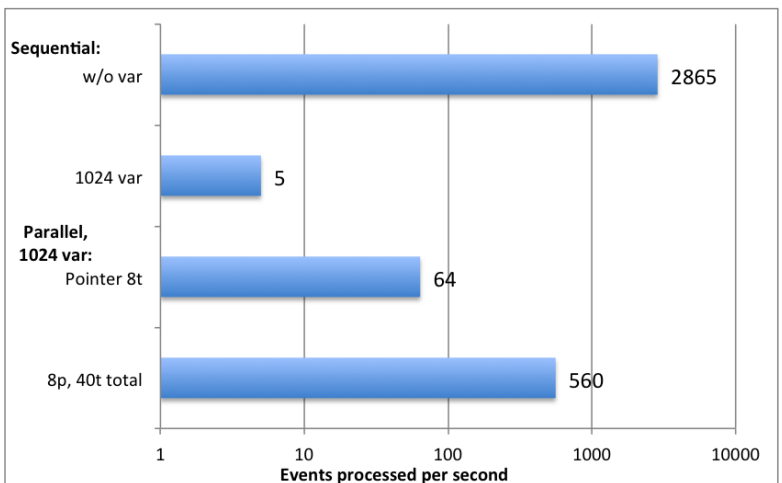

Fig. 11: Throughput of events processed for the original sequential ttH_dilep, with no and 1024 variations (var), and for the parallel pointer and multiprocess implementations (with $p$ processes and $t$ threads).

code but assuming an error of $\pm 1 \%$ on the detector measurements and considering 1024 variations on those values improving the analysis accuracy, (iii) a parallel single process implementation, with multiple threads, and (iv) the best parallel combination of processes and threads. By removing the code inefficiencies and developing efficient parallelisation strategies, the overall result of this work increased the event throughput by a factor of 112, from 5 to 560 events per second.

These promising results on code execution efficiency leave yet some room for further enhancements. The scheduler could be improved to automatically predict the best process/thread configuration for each system by analysing a set of microbenchmarks or the application itself on a small input, and ultimately identify the best core affinity scheme. Also, the application efficiency could be improved using hardware accelerators, balancing the workload among accelerators and CPU devices in heterogeneous systems. The use of development frameworks for heterogeneous systems, such as StarPU [18], may further improve productivity and efficiency through aids in code parallelization and transparent workload distribution among multi-core devices and computing accelerators.

Acknowledgments. This work is funded by National Funds through the FCT Fundação para a Ciência e a Tecnologia (Portuguese Foundation for Science and Technology) within project PEst-OE/EEI/UI0752/2014, by LIP (Laboratório de Instrumentação e Física Experimental de Partículas), and the SeARCH cluster (REEQ/443/EEI/2005).

\section{References}

1. The ATLAS Collaboration: The ATLAS Experiment at the CERN Large Hadron Collider. Journal of Instrumentation 3(08) (2008) S08003 
Pereira, Onofre, and Proença

2. Aad, G., et al.: Observation of a new particle in the search for the Standard Model Higgs boson with the ATLAS detector at the LHC. Phys.Lett. B716 (2012) 1-29

3. Oliveira, V., Pina, A., Castro, N., Veloso, F., Onofre, A.: Even Bigger Data: Preparing for the LHC/ATLAS Upgrade. 6th Iberian Grid Infrastructure Conference (2012)

4. Pereira, A.: Efficient Processing of ATLAS Events Analysis in Homogeneous and Heterogeneous Platforms. Master's thesis, University of Minho (September 2013)

5. F. Rademakers and P. Canal and B. Bellenot and O. Couet and A. Naumann and G. Ganis and L. Moneta and V. Vasilev and A. Gheata and P. Russo and R. Brun: ROOT (November 2012)

6. Graham, S.L., Kessler, P.B., Mckusick, M.K.: Gprof: A Call Graph Execution Profiler. SIGPLAN Not. 17(6) (June 1982) 120-126

7. Developers, V.: Callgrind: a call-graph generating cache and branch prediction profiler (January 2013)

8. Intel: Profiling Runtime Generated and Interpreted Code with Intel VTune Amplifier. Technical report (January 2013)

9. Browne, S., Deane, C., Ho, G., Muccima, P.: PAPI: A Portable Interface to Hardware Performance Counters. Proceedings of Department of Defense HPCMP Users Group Conference (June 1999)

10. Intel: Intel Xeon Processor E5 v2 Family: Datasheet. Technical report (September 2013)

11. Matsumoto, M., Saito, M.: Mersenne Twister: A 623-dimensionally equidistributed uniform pseudorandom number generator. ACM Transactions on Modeling and Computer Simulations: Special Issue on Uniform Random Number Generation (1998)

12. Board, O.A.R.: OpenMP Application Program Interface. Technical report (July 2013)

13. Dow, E.: Take charge of processor affinity. IBM developerWorks (September 2005)

14. Blackford, L.S., Demmel, J., Dongarra, J., Duff, I., Hammarling, S., Henry, G., Heroux, M., Kaufman, L., Lumsdaine, A., Petitet, A., Pozo, R., Remington, K., Whaley, R.C.: An Updated Set of Basic Linear Algebra Subprograms (BLAS). ACM Trans. Math. Soft., 28-2 (2002)

15. Corporation, I.: Intel 64 and IA-32 Architectures Optimization Reference Manual. Technical report, Intel Corporation (2013)

16. Corporation, I.: Intel 64 and IA-32 Architectures Software Developer's Manual. Technical report, Intel Corporation (February 2014)

17. Ott, D.: Optimizing Applications for NUMA. Technical report (February 2011)

18. Augonnet, C., Thibault, S., Namyst, R., Wacrenier, P.A.: Starpu: A unified platform for task scheduling on heterogeneous multicore architectures. Concurr. Comput. : Pract. Exper. 23(2) (February 2011) 187-198 